\title{
George Sand, écritures et représentations, textes réunis par Eric Bordas
}

\section{Annarosa Poli}

\section{(2) OpenEdition}

1 Journals

\section{Edizione digitale}

URL: http://journals.openedition.org/studifrancesi/34917

DOI: 10.4000/studifrancesi.34917

ISSN: 2421-5856

\section{Editore}

Rosenberg \& Sellier

\section{Edizione cartacea}

Data di pubblicazione: 1 novembre 2005

Paginazione: 433-434

ISSN: 0039-2944

\section{Notizia bibliografica digitale}

Annarosa Poli, «George Sand, écritures et représentations, textes réunis par Eric Bordas», Studi Francesi [Online], 146 (XLIX | II) | 2005, online dal 30 novembre 2015, consultato il 19 avril 2021. URL: http:// journals.openedition.org/studifrancesi/34917 ; DOI: https://doi.org/10.4000/studifrancesi.34917

Questo documento è stato generato automaticamente il 19 avril 2021.

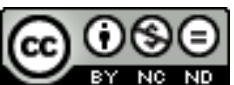

Studi Francesi è distribuita con Licenza Creative Commons Attribuzione - Non commerciale - Non opere derivate 4.0 Internazionale. 


\title{
George Sand, écritures et représentations, textes réunis par Eric Bordas
}

\author{
Annarosa Poli
}

\section{NOTIZIA}

AA. VV., George Sand, écritures et représentations, textes réunis par ERIC BORDAS, Paris, Eurédit, 2004, pp. 338.

1 Se i romanzi di George Sand oggi presentano delle difficoltà di lettura è perché sono in maggioranza «romans d'idées». Un gruppo di specialisti ha riletto la sua opera chiedendosi se il romanzo di George Sand è stato veramente un romanzo «a tesi».

2 Lucienne FRAPPIER-MAZUR ha analizzato con una metodologia particolarmente illuminante Mademoiselle La Quintinie, il romanzo dell'anticlericalismo (George Sand et le roman à thèse: autour de Mademoiselle La Quintinie, pp. 14-44) per distinguere l'espressione delle convinzioni della scrittrice dalla creazione fantastica nella struttura romanzesca. La prima constatazione è che questo romanzo si diversifica dagli altri come forma espressiva: il dialogo é polifonico e sostituisce il commento della voce narrante, tipico delle altre opere di George Sand. La romanziera è ormai settantenne, critica l'illusione romantica e ammira il progresso scientifico e industriale di quegli anni. Con un piglio più deciso di quello della Histoire de Sibylle di Octave Feuilllet, sotto la cui egida vuole inserirsi, si scaglia contro la deleteria influenza di un confessore cattolico su Mademoiselle La Quintinie che troverà più convincente le idee dell'uomo che ama, scegliendo il protestantesimo. Isabelle HOOG-NAGINSKI (George Sand et le réalisme prophétique, pp. 45- 66) esamina il Compagnon du tour de France, altro romanzo a sfondo sociale, con le stesse finalità di L. Frappier-Mazur, soffermandosi particolarmente sulla importanza della malinconia, male del secolo per la prima volta analizzato come disagio psicologico delle classi popolari insoddisfatte. Le Compagnon du tour de France con gli 
altri romanzi di ispirazione sociale (Horace, Jeanne, Isidora, $L a$ Ville noire) saranno condannati dalla critica. Le classi nobili, borghesi e il clero mal sopportarono che la scrittrice mescolasse il realismo balzachiano con l'idealismo «visionario» che le era proprio e soprattutto che trasportasse nella sua epoca personaggi di estrazione popolare, considerati nullità, ma che saranno più reali in un'epoca successiva quando sarà loro dato di manifestare tutte le loro qualità. Questo «realismo profetico», di cui la romanziera è profondamente convinta («l'homme tel qu'il devrait être») prepara una «révolution pour les moeurs futures» nella società e per conseguenza nella letteratura. Anne E. MCCALL-SAINT-SAENS (Savoir spéculatif et Littérature industrielle: Le Péché de M. Antoine et La Ville noire) e Nicole MOZET (Les mariages paysans dans l'œeuvre de George Sand: de Valentine à Marianne. Mésalliance, désir et vertu, p. 83-102) hanno scelto due argomenti di notevole rilievo politico e sociale: l'industrializzazione e il matrimonio con una persona di condizione inferiore. La prima conclude affermando che: «dans l'œuvre sandienne, où, il est vrai, la spéculation sur la perte n'appartient qu'au passé, celle-ci n'en constitue pas moins une puissante théorisation économique de la foi et du don, à l'âge du matérialisme capitaliste» e la seconda termina la sua indagine sostenendo che nei romanzi a sfondo campestre di George Sand: «Se marier consiste moins à abolir les différences qu'à trouver les ressemblances: c'est le triomphe de la vertu, ou, si l'on préfère d'une certaine dose d'équilibre physique comme constituant l'amour conjugal». Éléonore REVERZY conclude questa prima sezione proponendo un paragone tra Sand e Zola «Sand et Zola. Littérature et valeurs», pp. 103-119) due scrittori impegnati e due universi differenti, ma con simili intenti didattici per «educare i lettori»: George Sand tramite la forma della parabola e Zola quella della satira.

3 Questa riflessione su un punto particolare della poetica sandiana è ampliata nella seconda sezione («Lectures») letture più distaccate dalla tematica che si era imposta la ricerca di gruppo, ma resta pur sempre un filo conduttore legato alla persistente tendenza di George Sand a finalità didattiche e teologiche. Aimée Boutin (Indiana au pays des hommes: narration et désir dans le roman de George Sand, pp. 123-133) vede in Indiana un romanzo di omosocialità che lascia un po' al margine la rappresentazione della donna per mettere in primo piano i rapporti della coppia Ralph-Raymond. Pierre LAFORGUE (Lélia, poésie, philosophie et érotique de la désespérance en 1830, pp. 135-148) mette in luce l'importanza del riferimento storico al 1830 per meglio comprendere un romanzo come Lélia. David A. POWELL (Menteurs et mensognes dans Ma soeur Jeanne, pp. 149-167) propone una lettura tematica della menzogna che rivela una dialettica della ragione implicita nella enunciazione profonda del racconto. Gérard GENGEMBRE (Roman familial, Révolution et empire dans Histoire de ma vie, pp. 169-182) scopre un altro romanzo nella lettura di Histoire de ma vie e dimostra come i riferimenti storici della Rivoluzione e dell'Impero sono le basi portanti sia dell'immaginario sandiano che del piano generale dell'autobiografia. Olivier BARA (Représentations sandiennes du public de théâtre: la communauté impossible, pp. 183-206) ritorna ai romanzi, ma per soffermarsi sulla rappresentazione del teatro nei racconti, in particolare i romanzi degli artisti. Il pubblico dotato di preparazione, precedentemente educato, incarna una comunità ideale non senza ambiguità, il che ci riporta alla convinzione di George Sand che tutto è politica. Marie-Ève THERENTY (Songez que je n'ai aucune des conditions du journalisme, pp. 207-238) fa il punto sulla scrittura giornalistica della romanziera, aspetto molto spesso trascurato e invece importante in questa donna eclettica. 
4 Il volume si conclude con un «dossier» realizzato da Sabine LoucIF e David PowelL, ambedue docenti in Università americane, i quali propongono una bibliografia (1990-1999) frutto di dieci anni di ricerche sandiane in tutto il mondo, accompagnata da un'analisi critica della fortuna della romanziera nei singoli paesi, redatta con una metodologia esemplare per chiunque volesse seguirne l'esempio con altri autori. Per numero di studi gli Stati Uniti hanno la priorità, seguiti dalla Francia. In Europa seguono Inghilterra, Belgio, Germania, Olanda, Ungheria, Finlandia, Norvegia, Polonia, Spagna, ecc. Anche l'Italia, nei nove anni presi in considerazione, fa la sua figura per la pubblicazione nel 1994, di George Sand et son temps. Hommage à Annarosa Poli (ed. CIRVI Moncalieri) con ben quattro pagine (pp. 335-338) e un commento molto lusinghiero a p. 247. Peccato che nella bibliografia non compaia l'opera di Roberto Cuppone L'Invenzione della commedia dell'arte. Le Théâtre de Nohant, Moncalieri, CIRVI, 1997, coll. «Civilisation de l'Europe».

5 Questo volume si rivela di una straordinaria utilità per gli studiosi di George Sand sia per la tesi che vuole dimostrare sia per la singola bibliografia relativa a diversi articoli e per quella generale. 